\title{
Metastatic Breast Cancer as a Chronic Disease: Evidence-Based Data on a Theoretical Concept
}

\author{
Constanze Elfgen ${ }^{a, b}$ Giacomo Montagna ${ }^{c, d}$ Seraina Margaretha Schmid ${ }^{d, e}$ \\ Walter Bierbauer ${ }^{f}$ Uwe Güth ${ }^{\mathrm{a}} \mathrm{d}$ \\ ${ }^{a}$ Department of Breast Surgery, Brust-Zentrum Zürich, Zurich, Switzerland; ${ }^{b}$ Department of Senology,

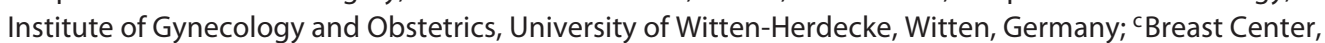 \\ University Hospital Basel, Basel, Switzerland; ${ }^{d}$ Department of Gynecology and Obstetrics,

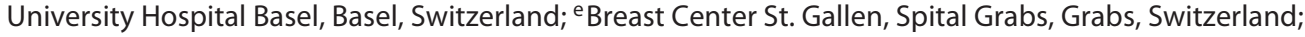 \\ ${ }^{f}$ Department of Psychology, Applied Social and Health Psychology, University of Zurich, Zurich, Switzerland
}

\section{Keywords}

Metastatic breast cancer - Chronic disease .

Noncompliance · Long-term survival · Patient-physician communication

\begin{abstract}
Background: We challenge the concept of metastatic breast cancer (MBC) as a chronic disease. Methods: We analyzed an unselected cohort of 367 patients who were diagnosed with MBC over a 22-year period (1990-2011). Results: In order to create a "chronic disease subgroup", we separated those patients from the entire cohort in whom systemic therapy was not applied after the diagnosis of MBC $(n=53 ; 14.4 \%)$. Three hundred fourteen patients (85.6\%) comprised the "chronic disease subgroup". The vast majority of those patients (89.8\%) died of progressive disease after a median metastatic disease survival (MDS) of 25 months. Twenty patients (6.4\%) died of non-MBC-related causes (MDS 38.5 months). Approximately 1 in 4 patients (26.8\%) died within the first year after the MBC diagnosis. The 3- and 5-year MDS rates were 35.4 and $16.2 \%$, respectively. Only 12 patients (3.8\%) were exceptional survivors (MDS >10 years). Conclusion: The term "chronic disease" might be appropriate in selected MBC cases, bringing MBC into alignment with "classical" chronic diseases such as diabetes and hypertension. However, most cases display fundamental differences with re-
\end{abstract}

gard to temporal progression and above all the case fatality rate. More than $90 \%$ of patients in the "chronic disease subgroup" died of the disease with a MDS of 2-3 years (even those who underwent systemic palliative therapies). Doctors and patients might understand the term "chronic disease" differently. The term must be used sparingly and explained carefully in order to create a common level of communication based on a shared understanding which avoids awakening false hopes and fostering misleading expectations.

C 2019 S. Karger AG, Basel

\section{Introduction}

Distant metastatic breast cancer (MBC) is generally considered to be a treatable, but not curable, condition [1]. However, through the introduction of a new generation of effective agents with safer profiles, together with considerable advances in supportive care, longer survival times have been achieved during the last 2 decades, resulting in an increasing number of women living with $\mathrm{MBC}$ [2]. As a result of this positive development, one of the memes now embraced by both physicians and patients is that of "metastatic cancer as a chronic disease" [3-6].

The term "chronic disease" is applied to a wide range of conditions [7]. In developed countries, in addition to cardiovascular diseases (e.g., high blood pressure), dia- 
betes, obesity, arthritis, neurologic diseases, and respiratory ailments such as bronchial asthma and chronic obstructive pulmonary disease (COPD), cancer is also considered as one of the leading chronic disorders [813]. The list of chronic diseases is very heterogeneous, and each disease has considerable variability in its degree of severity and its course. This makes it difficult to generalize the therapeutic approach. One main therapeutic task in the management of chronic diseases is fulfilled when a disease that cannot be completely cured can be treated with continuous or periodic therapy that controls its progression and alleviates its symptoms with limited toxicity. One could argue that metastatic cancer and chronic diseases share this general therapeutic principle.

The concept of $\mathrm{MBC}$ as a chronic disease is widely debated not only by physicians but also in many patient blogs. However, more and more critical objections are being made that the label "chronic disease" is inappropriate for MBC [3-6]. In fact, the term "chronic disease" used in discussions between physicians and patients diagnosed with $\mathrm{MBC}$ poses risks in that a common level of communication based on a shared language (and a shared understanding of key terms) is no longer applicable [7]. It may give patients the idea that they do not have a life-threatening and in most cases also a lethal condition.

In this study we challenge the concept of $\mathrm{MBC}$ as a chronic disease. Evidence-based data which might elucidate this concept is scarce. This deficit arises from the relative absence in the $\mathrm{MBC}$ literature of robust data with respect to the following 2 factors which are essential prerequisites for such an analysis:

1. Reliable data collection on the long-term survival of MBC patients.

2. Noncompliance on systemic palliative therapy. One main inclusion criterion for a chronic disease process is that its progress can be controlled or stabilized by continuous or periodic systemic therapy. In this study, we established a "chronic disease subgroup" by discounting from the full study cohort those patients who did not undergo systemic therapy after the diagnosis of MBC.

Based on a prospectively maintained breast cancer database which includes all newly diagnosed cases at a large Swiss breast center over a 20-year period (1990-2009), we analyzed an unselected, consecutive cohort of MBC patients. Since our data covers an observation period of almost 30 years, we are able to report the frequency of the occurrence of long-term MBC survivors. Additionally, we can provide information about a subgroup of patients which is commonly underreported in the oncologic literature, i.e., those patients who, for various reasons, do not receive palliative systemic therapy.

\section{Patients and Methods}

Data from the prospective relational Basel Breast Cancer Database (BBCD), which includes all newly diagnosed primary invasive breast cancer cases treated at the University Women's Hospital Basel (Switzerland) since 1990, provided the basis for this study. For this study, data from all female patients who were diagnosed with breast cancer up to and including 2009 was analyzed $(n=$ $1,459)$.

During this 20 -year period, 92 patients $(6.3 \%)$ had primary MBC at diagnosis (stage IV). In 2011, with the exception of 37 patients (2.5\% of the entire cohort), who were lost to follow-up after a median follow-up time of 36 months (range 1-166), outcome information was available for all patients recorded in the BBCD. As of March 2011, two hundred seventy-eight patients (20.3\% of all patients who had stage I-III disease at initial diagnosis) had developed secondary distant metastases over time. Ultimately, the entire cohort of women with confirmed MBC consisted of 370 patients. Three patients were not considered for analysis because we did not have reliable information about their distant recurrent-free survival times and their palliative systemic therapies (overall survival of these patients: 25,85 , and 116 months, respectively).

We recorded the general type of therapy during the course of the disease. Furthermore, the reasons that systemic therapy was not carried out were found to be as follows:

- Noncompliance: the patient did not start any palliative systemic therapy that was on offer or had been recommended. In this study, we generally defined "noncompliance" as the unwillingness of the patient to accept starting any palliative systemic therapy that was on offer or had been recommended. We not only defined those patients who decided not to start any therapy as "noncompliant" but also extended this definition on patients who stopped therapy after a short time, that is, after receiving only one cycle of chemotherapy or after the intake of $<$ 2 weeks of endocrine therapy.

- Not recommended: patients who did not receive a recommendation to undertake systemic palliative therapy for various medical reasons.

- Acute medical events: systemic therapy could not be started as a result of unexpected internal medical or MBC-related events. Out of the 367 patients who were ultimately included in the study cohort, 355 patients (96.7\%) were followed until death; in these cases, the year of and age at death and the cause of death were recorded. Two patients $(0.5 \%)$ were lost to follow-up (metastatic disease survival [MDS] at time of last consultation: 16 and 61 months, respectively). Ten patients $(2.7 \%)$ who remained alive were followed until November/December 2018 (median MDS at the time of the last follow-up: 174 months, range 108-300).

In all but 4 cases, information regarding the year of and age at $\mathrm{MBC}$ diagnosis, the location of the first metastatic lesion, and the number of metastatic sites was recorded. In all but 5 cases we had complete information regarding adjuvant and palliative therapy courses.

\section{Statistical Analysis}

Using the Kaplan-Meier method, MDS was calculated from the date of diagnosis of MBC to the date of death or, for patients who survived, the date of last follow-up. Statistical differences between groups in terms of survival curves were analyzed using the logrank test. To compare ordinal variables between 2 groups, the nonparametric Wilcoxon test was performed. To compare ordinal variables between more than 2 groups, the nonparametric Kruskal-Wallis test was performed. Comparisons between nominal parameters were made with Fisher's exact test. $p<0.05$ was considered statistically significant. 
Table 1. Clinicopathologic characteristics of 367 patients with MBC (entire cohort)

\begin{tabular}{|c|c|c|c|c|}
\hline Variable & $\begin{array}{l}\text { Entire cohort } \\
(n=367)\end{array}$ & $\begin{array}{l}\text { No systemic } \\
\text { therapy }(\mathrm{A}) \\
(n=53)\end{array}$ & $\begin{array}{l}\text { Chronic disease } \\
\text { subgroup (B) } \\
(n=314)\end{array}$ & $\begin{array}{l}p \text { value } \\
\text { A vs. B }\end{array}$ \\
\hline Age at diagnosis, years & $64(28-94)$ & $78(30-94)$ & $62(28-92)$ & $<0.001$ \\
\hline \multicolumn{5}{|l|}{ Year of MBC diagnosis } \\
\hline 1990-1999 & $145(39.5)$ & $18(34.0)$ & $127(40.4)$ & \multirow[t]{2}{*}{0.45} \\
\hline 2000-2011 & $222(60.5)$ & $35(66.0)$ & $187(59.6)$ & \\
\hline \multicolumn{5}{|l|}{ TNM stage at the initial diagnosis } \\
\hline Stage IV & $91(24.8)$ & $4(7.5)$ & $87(27.7)$ & \multirow[t]{2}{*}{0.001} \\
\hline \multicolumn{4}{|l|}{ Hormonal receptor status ${ }^{1}$} & \\
\hline Positive & $268(74.4)$ & $27(51.9)$ & $241(78.2)$ & \multirow{3}{*}{$<0.001$} \\
\hline Negative & $92(25.6)$ & $25(48.2)$ & $67(21.8)$ & \\
\hline Not available & 7 & 1 & 6 & \\
\hline Her2 status ${ }^{1,2, \mathrm{n}}$ & 113 & 17 & 96 & \\
\hline Positive & $30(26.5)$ & $4(23.5)$ & $26(27.1)$ & \\
\hline Triple negative & $21(18.6)$ & $8(47.5)$ & $13(13.5)$ & NA \\
\hline Not available & - & - & - & \\
\hline \multicolumn{5}{|l|}{$\begin{array}{l}\text { Pattern of metastases at the time } \\
\text { of MBC diagnosis }\end{array}$} \\
\hline Visceral metastases & $260(71.4)$ & $37(71.2)$ & $223(71.5)$ & \multirow[t]{3}{*}{1.00} \\
\hline Bone only & $103(28.3)$ & $14(26.9)$ & $89(28.5)$ & \\
\hline Unknown & 3 & 1 & 2 & \\
\hline Solitary metastatic organ site & $207(57.0)$ & $25(48.1)$ & $182(58.3)$ & 0.18 \\
\hline \multicolumn{5}{|l|}{ Metastatic sites } \\
\hline Bone & $226(62.1)$ & $34(65.4)$ & $192(61.5)$ & 0.65 \\
\hline Lung & $137(37.6)$ & $25(48.1)$ & $112(35.9)$ & 0.12 \\
\hline Liver & $92(25.3)$ & $18(34.6)$ & $74(23.7)$ & 0.12 \\
\hline Lymph nodes ${ }^{3}$ & $88(24.2)$ & $8(15.4)$ & $80(25.6)$ & 0.11 \\
\hline Brain & $24(6.6)$ & $11(21.2)$ & $13(4.2)$ & $<0.001$ \\
\hline Others & $36(9.9)$ & $4(7.7)$ & $32(10.3)$ & 0.80 \\
\hline
\end{tabular}

Comparison between patients without systemic palliative therapy (subgroup A) and patients who received systemic palliative therapy (chronic disease subgroup). Values are presented as means \pm SD or medians (range). Bold indicates a statistically significant difference $(p<0.05)$. NA, not available.

${ }^{1}$ Hormonal receptor status and HER2 status were measured in the primary breast tumor.

${ }^{2}$ Because HER-2 status has been routinely assessed for all patients since 2002, we included data from 2002 to 2009 (only in the analysis of this particular characteristic).

${ }^{3}$ Other than ipsilateral BC-related locoregional lymph nodes.

\section{Results}

The clinicopathology, treatment, and outcome characteristics of the 367 patients with MBC included in this study are summarized in Tables 1 and 2.

\section{Criteria for a "Chronic Disease Subgroup"}

The "chronic disease subgroup" on which our analysis was based was a cohort consisting of those patients who received systemic therapy after the diagnosis of MBC. It was created from the full cohort by discounting those patients who did not receive such treatment. Figure 1 illustrates the paths of this selection process. After careful analysis of the disease and therapy courses, 314 patients $(85.6 \%)$ comprised the "chronic disease subgroup" (Tables 1,2 , subgroup B). In total, 53 cases (14.4\% of the en- tire study cohort, see subgroup A) were not included in the "chronic disease subgroup". The reasons why systemic palliative therapy was not carried out are as follows (Fig. 1):

- Noncompliance $(n=42)$. The median age of this subgroup was 69 years (range 44-94), and the median MDS was 2.5 months (range 0.5-69).

- Not recommended $(n=7)$. The median age of this subgroup was 88 years (range 78-91), and the median MDS was 2.5 months (range 1-16).

- Acute medical events $(n=4)$.

The frequency of patients who, for various reasons, did not receive systemic therapies after MBC diagnosis was equally distributed over time (1990-1999: $12.4 \%$ vs. 2000-2011: 15.8\%, $p=0.45$ ). 
Table 2. Therapy and outcome characteristics

\begin{tabular}{|c|c|c|c|c|}
\hline Variable & $\begin{array}{l}\text { Entire cohort } \\
(n=367)\end{array}$ & $\begin{array}{l}\text { No systemic } \\
\text { therapy }(\mathrm{A}) \\
(n=53)\end{array}$ & $\begin{array}{l}\text { Chronic disease } \\
\text { subgroup (B) } \\
(n=314)\end{array}$ & $\begin{array}{l}p \text { value } \\
\text { A vs. B }\end{array}$ \\
\hline \multicolumn{5}{|l|}{ Previous systemic therapy } \\
\hline None (therapy naive) & $147(40.1)$ & $17(32.1)$ & $130(41.5)$ & 0.23 \\
\hline Unknown & 1 & - & 1 & \\
\hline \multicolumn{5}{|l|}{ Palliative systemic therapy, $n(\%)$} \\
\hline None & $55(15.0)$ & - & $2(0.6)$ & NA \\
\hline ET alone & $85(23.2)$ & 4 & $85(27.2)$ & \\
\hline $\mathrm{CT}^{1}$ alone & $85(23.2)$ & 3 & $85(27.2)$ & \\
\hline Combined therapy: ET $+\mathrm{CT}^{1}$ & $141(38.5)$ & - & $141(45.0)$ & \\
\hline Unknown regimen & 1 & - & 1 & \\
\hline Therapy lines, $n$ & $3(1-13)$ & - & $3(1-13)$ & \\
\hline \multicolumn{5}{|l|}{ Outcome status } \\
\hline Died on $\mathrm{MBC}$ & $333(90.7)$ & $51(96.2)$ & $282(89.8)$ & 0.20 \\
\hline Died of other causes & $22(6.0)$ & $2(3.8)$ & $20(6.4)$ & \\
\hline Alive (metastatic disease) $)^{2}$ & $5(1.4)$ & - & $5(1.6)$ & \\
\hline Alive (no evidence of disease) $)^{2}$ & $7(1.9)$ & - & $7(2.2)$ & \\
\hline \multicolumn{5}{|l|}{ Survival time $e^{2}$} \\
\hline DRFS, months & $38.5(2-215)$ & $39(6-184)$ & $38.5(2-215)$ & 0.72 \\
\hline MDS, months & $21(0.5-300)$ & $2(0.5-69)$ & $26.5(1-300)$ & $<0.001$ \\
\hline MDS $\leq 3$ months & $47(12.8)$ & $31(58.5)$ & $16(5.1)$ & NA \\
\hline MDS $\leq 12$ months & $130(35.4)$ & $46(86.8)$ & $84(26.8)$ & \\
\hline MDS $\geq 3$ years & $112(30.5)$ & $1(1.9)$ & $111(35.4)$ & \\
\hline MDS $\geq 5$ years & $52(14.2)$ & $1(1.9)$ & $51(16.2)$ & \\
\hline MDS $\geq 10$ years & $12(3.3)$ & - & $12(3.8)$ & \\
\hline Overall survival, months & $54(1-341)$ & $37(2-186)$ & $56.5(1-341)$ & 0.006 \\
\hline
\end{tabular}

The entire cohort consisted of 367 patients. Values are presented as means \pm SD, medians (range), or numbers (\%). Bold indicates a statistically significant difference $(p<0.05)$.

Subgroup A: patients who did not receive systemic palliative therapy. Subgroup B: "chronic disease subgroup".

${ }^{1}$ Includes also immunotherapy with trastuzumab.

${ }^{2}$ Two patients were lost to follow-up (MDS at the time of the last consultation: 16 and 61 months, respectively). The patient with the longer MDS had ovarian metastases at the initial MBC diagnosis; endocrine therapy was given for hormone receptor-positive disease; there was no evidence of disease at the last follow-up. MBC, distant metastatic breast cancer; DRFS, distant recurrence-free survival; ET, endocrine therapy; CT, chemotherapy; NA, not available.

\section{Comparison of Both Subgroups}

Compared with the "chronic disease subgroup", those who remained untreated were significantly older (78 vs. 62 years, $p<0.001)$, more often had an aggressive tumor subtype (hormone receptor-negative carcinomas: 48.2 vs. $21.8 \%, p<0.001)$ and secondary MBC ( 92.5 vs. $72.3 \%$, $p=0.001)$.

Between patients in the "chronic disease subgroup" and those who did not receive systemic therapy there were no significant differences with regard to the presence of visceral metastases $(71.5$ vs. $71.2 \%, p=1.00)$. We observed a lower percentage of multiple metastatic sites in the "chronic disease group", though this did not reach statistical significance ( $>1$ metastatic organ site: 41.7 vs. $51.9 \%, p=0.18)$. With regard to the distribution of visceral metastatic sites, the patients in whom no systemic therapy had been performed had significantly more frequent brain metastases $(21.2$ vs. $4.2 \%, p<0.001)$. While both subgroups were similar in terms of the interval from diagnosis of the primary disease to the time of MBC (DRFS: 39 vs. 38.5 months, $p=0.72$ ), survival times after MBC diagnosis were significantly shorter in the "no-therapy group" (MDS: 2 vs. 26.5 months, $p<0.001$ ).

\section{Outcome of the "Chronic Disease Subgroup"}

Figure 2 shows the survival curve of the entire cohort $(n=367)$ as well as the survival curve of the "chronic disease subgroup" $(n=314)$. It displays a shift in the MDS rates from 21 months (entire cohort) to 26.5 months ("chronic disease subgroup", $p=0.046$ ). Table 2 shows that approximately 1 out of 4 patients died within the first year after MBC diagnosis. The 3- and 5-year MDS were 35.4 and $16.2 \%$, respectively. Twelve patients $(3.8 \%)$ were exceptional survivors, with an MDS of more than 10 years.

Figure 3 shows survival times with regard to different clinicopathologic characteristics. Even in a subcohort of patients who displayed a combination of several favorable 


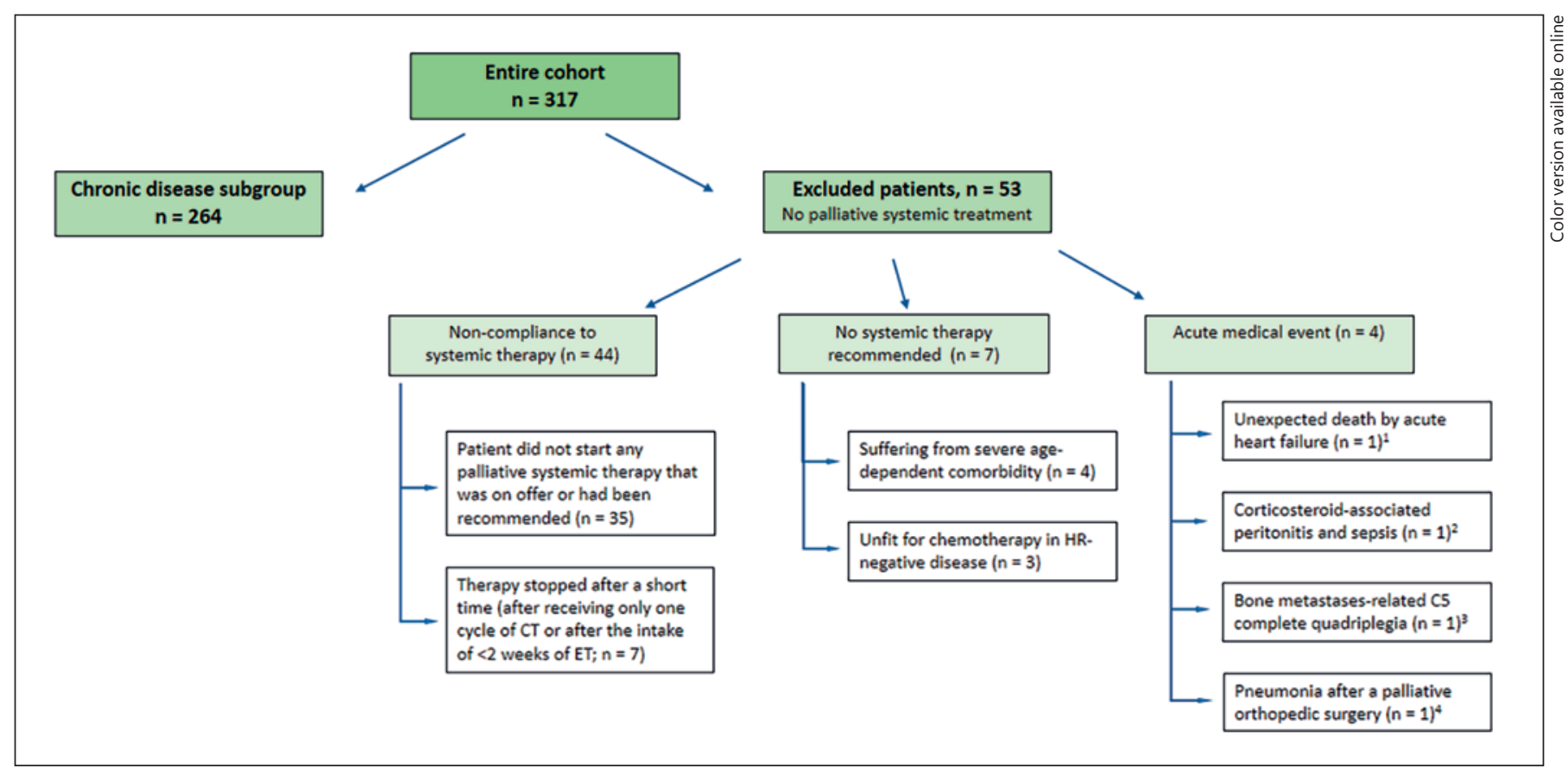

Fig. 1. Selection process of the "chronic disease subgroup". CT, chemotherapy; ET, endocrine therapy; HR, hormone receptor; MS, metastatic organ site. ${ }^{1}$ Age at MBC diagnosis: 79 years; MS: lymph nodes; MDS: 1 week. ${ }^{2}$ Age at MBC diagnosis: 30 years; MS: brain; palliative therapy: transsphenoidal pituitary tumor resec- tion; MDS: 4 weeks. ${ }^{3}$ Age at MBC diagnosis: 66 years; MS: bone (multiple), mediastinal lymph node; palliative therapy: anterior cervical corpectomy; MDS: 8 weeks. ${ }^{4}$ Age at MBC diagnosis: 93 years; MS: bone (multiple); palliative therapy: orthopedic surgery (pathological fracture of the hip); MDS: 1 week. clinicopathologic characteristics (a younger age, hormone receptor-positive disease, and only 1 metastatic organ site; $n=87 ; 27.7 \%$ of the "chronic disease subgroup") the median MDS was only 37 months.

At the time of data analysis in November/December 2018 , the vast majority of the patients $(89.8 \%)$ had died of progressive disease after a median MDS of 25 months (range 1-127) at a median age of 65 years (range 29-93). It is to be expected that this percentage will increase slightly up to approximately 91-92\%. Four long-term survivors $(1.3 \%)$ were still alive at the time of the last follow-up but suffered from progressive disease. These patients had a median MDS of 136.5 months (range 112$188)$ and were of an advanced age $(72,79,84$, and 85 years, respectively).

Twenty patients $(6.4 \%)$ died of other, non-MBC related causes. Those women had a median MDS of 38.5 months (range 3-126). The median age at diagnosis of MBC was 72.5 years (range 46-89) and the median age at death was 74.5 years (range 52-94). It is to be expected that this percentage will increase to approximately $8 \%$ due to 6 long-term survivors (1.9\%) who were not only alive at last follow-up but who had also exhibited no evidence of disease. These women had a median MDS of 139 months (range 108-300). The median age at diagnosis of $\mathrm{MBC}$ was 47 years (range 39-60).

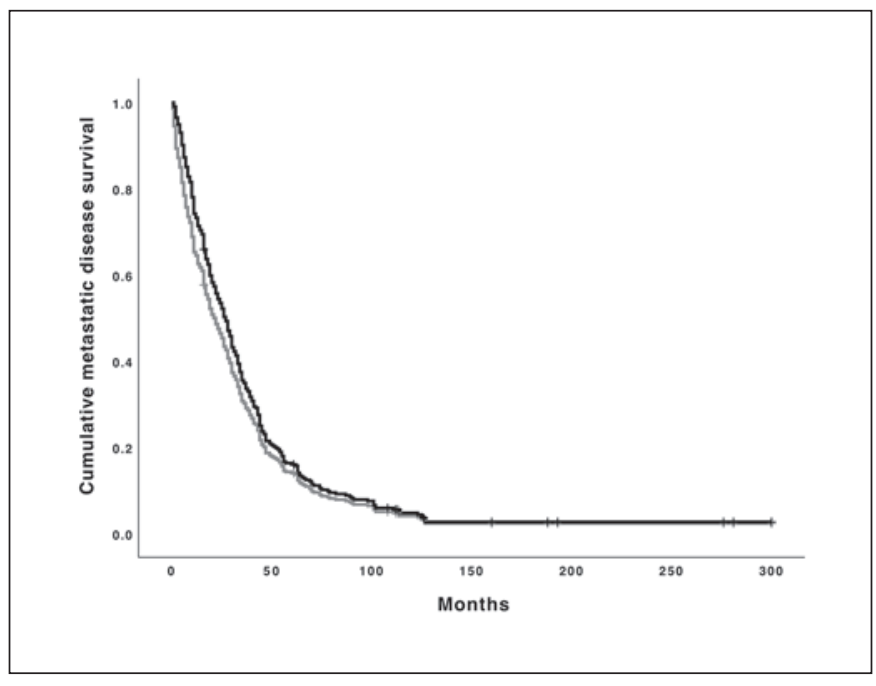

Fig. 2. Comparison of MDS of unselected patients with MBC (entire cohort, $n=367$, grey line) vs. the "chronic disease subgroup" ( $n=314$, black line); one-tailed test: $p=0.046$.

\section{Discussion}

Our study has some limitations. First, it only includes patients from a single region of a small country with a high socioeconomic status where all inhabitants have universal access to health care. Second, it is a retrospective 


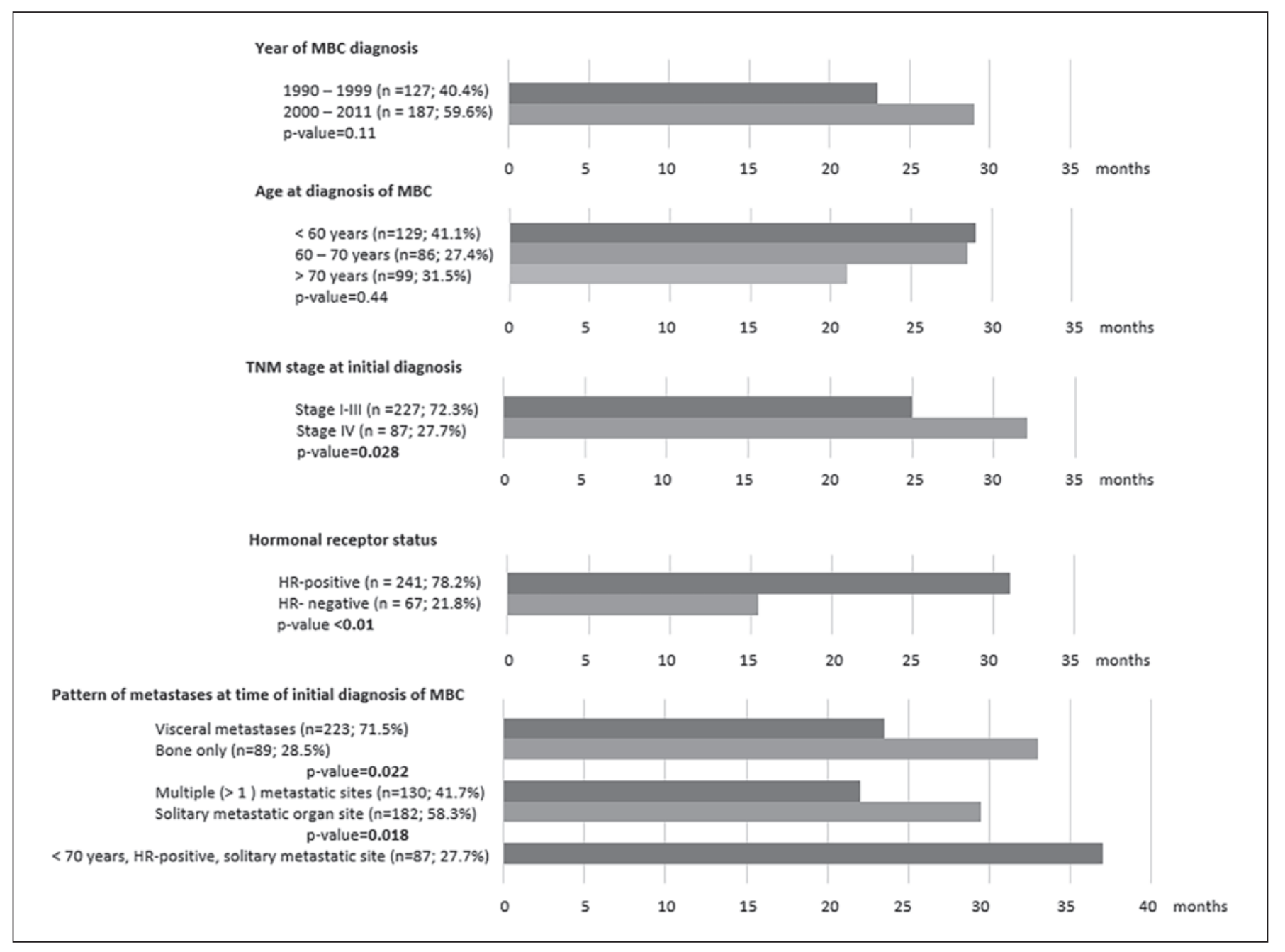

Fig. 3. MDS times with regard to different clinicopathologic characteristics. HR, hormone receptor.

analysis. However, this study has the important strength of an almost complete data documentation of:

- The entire cohort. Our prospectively maintained database included all patients newly diagnosed with breast cancer over a 20-year period (1990-2009). The rate of patients who were lost-to-follow-up was very low (< $3 \%$ ), and only very few patients, who could have potentially developed MBC, were missed.

- The group of patients who were diagnosed with MBC. The vast majority (>98\%) of the palliative courses were completely documented with regard to metastatic patterns and palliative therapy.

\section{Criteria for a "Chronic Disease Subgroup"}

In order to challenge the concept of $\mathrm{MBC}$ as a chronic disease, we created a "chronic disease subgroup". One main inclusion criterion for a chronic disease is that its progress can be controlled or stabilized by continuous or periodic systemic therapy. Due to the complete documentation of the entire cohort, we were able to separate those patients who received no systemic antineoplastic therapy during their palliative disease course. Consequently, approximately $14 \%$ of the patients of the entire cohort were excluded from the chronic disease subgroup; the majority of these patients $(83 \%)$ had actively decided against the recommended therapy and could therefore also be designated as a "noncompliance subgroup". Interestingly, there was no change in the frequency of occurrence of patients who did not receive palliative systemic therapy over time. It might have been assumed that with the new generation of effective agents with safer profiles and with considerable advances in supportive care (a development that started around the late 1990s) the reservations of patients about oncologic therapies would have decreased. That was not the case in our cohort.

Our model of data analysis could also be usefully applied in other retrospective analyses of large cancer cohorts. For a clear and comprehensive real-world picture of the effectiveness of palliative systemic therapy on MDS in an otherwise unselected cohort it is essential to take 
into account those patients who had undergone systemic therapy and to separate them from those patients who for whatever reason did not undergo systemic therapy. After creating this "chronic disease subgroup", we observed a marked shift in the MDS rates from 21 to 26.5 months.

\section{Is MBC a Chronic Disease?}

There can be no doubt that there are overlaps in definition between "classical" chronic diseases and metastatic cancer: they are both long-lasting or recurrent; they both require long-term treatment, supervision, observation or care; they are caused by nonreversible pathological alterations; and they can be altered but not cured by medication $[14,15]$. Furthermore, the general therapy principles of chronic disease (long-term stabilization with a good tolerance to drugs that have a limited cumulative toxicity) also correspond with those of palliative cancer therapy. On the other hand, the majority of chronic diseases and MBC display fundamental differences with regard to temporal progression and above all the case fatality rate.

The term "chronic" has its origin in Greek (khronikos, i.e., of time). In the particular case of illness, it now means in English "persisting for a long time or constantly recurring" [16]. The criteria and definitions of just how much time must pass before the term can legitimately be applied vary widely in medical practice. Often only general, nonspecific formulations are given, e.g., "persists for a long time" [10], "persistent or otherwise long-lasting" [11], or "of long duration and generally slow progression" [9]. The US Centers for Disease Control and Prevention (CDC) define chronic diseases as "conditions that last 1 year or more and require ongoing medical attention" [8]. The most commonly used marker for a chronic condition is a 3-month time span [11-13]. It is remarkable that this period is also used by the National Cancer Institute [12], even though in oncology and especially in relation to metastatic diseases this period makes very little sense. To exemplify this, in the case of progressive metastatic disease which leads to death within 6-12 months the definition period has been clearly exceeded, but the course of the disease "hardly seems to fall under what a reasonable person would think of as a chronic disease" [6].

A well-established parameter for estimating the prognosis of a particular disease is the 5-year survival rate. When this period is taken as the potential criterion for characterizing a disease as chronic, only $16 \%$ of the chronic disease subgroup patients of our study cohort achieved this survival time. Only approximately $4 \%$ of the patients had an MDS of more than 10 years. When after the diagnosis of $\mathrm{MBC}$ the doctor informs the patient of the prognosis, this long-term survival rate might arguably be presented as a prospective goal. However, it seems unjustifiable to represent the $\mathrm{MBC}$ condition as a whole as a chronic disease. The nature of MBC is clearly different from that of obesity, diabetes, high blood pressure, arthritis, or COPD. These diseases can lead to death, but not inevitably so. In contrast, more than $90 \%$ of the MBC patients die of the disease, with a survival time of 2-3 years after diagnosis of distant metastases - even those who undergo systemic palliative therapies.

\section{"MBC Is Like Diabetes:" the Term "Chronic Disease" as a Potential Source of Misinterpretation in Patient- \\ Physician Communication}

It is one of the most unpleasant and difficult tasks for a doctor to have to explain to a patient that they are suffering from an incurable metastatic disease which, despite therapy, will in the normal course of events result in their death within 2-5 years. The physician has the important task of providing a clear and sensitive prognosis while building the courage and the confidence to face the coming course of the disease. It may be superficially attractive to designate the further course of the disease as "chronic", but it should not surprise the doctor that a patient understands the term "chronic disease" differently [7]. The median age of the patients of our "chronic disease subgroup" was 62 years; approximately one third of the patients were older than 70 years. The majority of the women at this age had already been confronted with one or more chronic diseases with which they have learned to cope over many years $[8,10,11]$. The statement by the doctor that the MBC constitutes just one more chronic disorder will not be particularly unsettling for many patients. The term "chronic disease" might be misleading because patients might interpret that MBC could be controlled by long-term disciplined care similarly to diabetes or hypertension [7]. Patients may even draw a comparison to AIDS in that new cancer drugs might be as successful as the antiretroviral therapy which transformed this disease from an almost invariably fatal infection into a manageable chronic condition that for many will span several decades of life [17]. Because of this, the danger exists of departing from a common level of communication based on a shared language and understanding: using the term "chronic disease" might awaken false hopes and misleading expectations that patients do not have a lethal disease $[3,4]$ - which is clearly not the case.

A misleading message might result in a high prognosis discordance between the patient and the oncologist. Gramling et al. [18] reported this discordance in approximately $70 \%$ of cases. In that study patients with advanced cancer estimated their 2-year survival probability without exception more favorably than their physicians did. Ninety percent of the patients were not aware that their oncologists held a much more skeptical prognosis than they did but believed that their doctors shared their own positive viewpoint. If a patient with advanced cancer expects with $90-100 \%$ certainty to survive more than 2 years when 
at the same time the doctor estimates the 2-year survival as $10-20 \%$, this can be problematic for the further course of the disease because the patient's unrealistic perceptions of the survival prognosis might lead to inappropriate therapies with a questionable impact on survival.

Nevertheless, there are situations in which doctors may feel the need to use a term such as "chronic disease", but in this situation it must be used sparingly and explained carefully in order to avoid miscommunication [7]. Numerous studies have shown that advanced cancer patients and their families, but also their doctors, avoid delicate issues concerning the limitation on life and the prognosis of survival time [19]. The term "chronic" could lead to a trivialization of the disease and might then become part of a mutual avoidance strategy.

It is probably better to avoid describing a complex, multifaceted disease such as MBC with a single term. In each individual case we have to ask ourselves what terminology can be appropriately used to describe the disease progress and prognosis of a patient who has just been diagnosed with MBC and who:

- is living with progressive disease, that

- will lead in the vast majority of cases to death, but who

- is clearly not in the terminal phases of illness and who

- may receive targeted cancer treatment which is often able to stabilize the disease over several years with tolerable toxicity.
The term "chronic disease" appears inappropriate in this situation. We must confront this terminological problem. Particularly at the end of life an appropriate patient-physician communication in a mutually understood terminology is essential to achieve an oncologic surveillance that honors a patient's values, preferences, and wishes $[18,19]$.

\section{Statement of Ethics}

The study design and data collection methods were approved by the institutional review board.

\section{Disclosure Statement}

The authors have no conflict of interests to declare.

\section{Author Contributions}

Prof. Uwe Güth had full access to all of the data in the study and takes responsibility for the integrity of the data and the accuracy of the data analysis. All of the authors directly participated in the planning of the study concept, analysis and interpretation of the data, and drafting and critical revision of this paper. They read and approved the final version submitted for publication.

\section{References}

1 Cardoso F, Senkus E, Costa A, Papadopoulos E, Aapro M, André F, et al. 4th ESO-ESMO International Consensus Guidelines for Advanced Breast Cancer (ABC 4). Ann Oncol. 2018 Aug;29(8):1634-57.

2 Mariotto AB, Etzioni R, Hurlbert M, Penberthy L, Mayer M. Estimation of the Number of Women Living with Metastatic Breast Cancer in the United States. Cancer Epidemiol Biomarkers Prev. 2017 Jun;26(6):809-15.

3 Brenner BA. Treating breast cancer as a recurrent-not chronic-disease. San Francisco (CA): Breast Cancer Action [Internet]. Posted on March 21, 2009 [cited 2019 Apr 10]. Available from: https://bcaction.org/2009/ $03 / 21 /$ treating-breast-cancer-as-a-recurrent $\%$ E $2 \% 80 \% 94$ not-chronic. \% E2 \% $80 \%$ 94disease

4 O’Brien K. Please Stop Calling Metastatic Breast Cancer a Chronic Disease [Internet]. Posted on July 30, 2018 [cited April 5 2019). Available from: https://www.linkedin.com/ pulse/please-stop-calling-metastatic-breastcancer-chronic-disease-o-brien/.

5 Sledge GW Jr. Musings of a Cancer Doctor: chronic. Oncology Times. 2014;36(20):7-11.

6 Sledge GW Jr. Curing Metastatic Breast Cancer. J Oncol Pract. 2016 Jan;12(1):6-10.
7 Bernell S, Howard SW. Use Your Words Carefully: What Is a Chronic Disease? Front Public Health. 2016 Aug;4:159.

8 About chronic diseases [Internet]. Druid Hills (GA): Centers for Disease Control and Prevention (CDC); 2018 Mar 8 [cited 2019 Apr 10]. Available from: https://www.cdc. gov/chronicdisease/about/index.htm.

9 Noncommunicable Diseases [Internet]. Geneve, Switzerland: World Health Organization (WHO); 2019 Jan 10 [cited 2019 Apr 10]. Avaialble from: https://www.who.int/ncds/ en/.

10 Shiel WC Jr. Medical definition of chronic disease [Internet]. Atlanta (GA): MedicineNet; 2018 Dec 21 [cited 2019 Apr 10]. Available at: https://www.medicinenet.com/script/ main/art.asp?articlekey $=33490$.

11 Chronic condition [Internet]. San Francisco (Ca): Wikipedia; 2019 Apr 10 [cited Apr 2019 10]. Available at: https://en.wikipedia.org/ wiki/Chronic_condition.

12 NCI Dictionary of Cancer Terms: chronic disease [Internet]. Maryland (MD): National Cancer Institute (NCI); 2019 Apr 10 [cited 2019 Apr 10]. Available at: https://www.cancer.gov/publications/dictionaries/cancerterms/def/chronic-disease.
13 About chronic conditions [Internet]. Washington, DC: National Health Council; 2016 Mar 21 [cited 2019 Apr 10]. Available at; http://www.nationalhealthcouncil.org/newsroom/about-chronic-conditions.

14 Lubkin I, Larsen P. Chronic Illness. Sudbury (MA): Jones \& Bartlett; 2002.

15 Norris S, Glasgow R, Engelgau M, et al. Chronic disease management: a definition and systematic approach to component interventions. Dis Manag Health Outcomes. 2003 Oct;11(8):477-88.

16 Definition of "chronic" in English [Internet]. Oxford (UK): In Dictionary. Oxford English Dictionary; 2019 Apr 10 (cited 2019 Apr 10). Available at: https://en.oxforddictionaries. com/definition/chronic.

17 Deeks SG, Lewin SR, Havlir DV. The end of AIDS: HIV infection as a chronic disease. Lancet. 2013 Nov;382(9903):1525-33.

18 Gramling R, Fiscella K, Xing G, Hoerger M, Duberstein P, Plumb S, et al. Determinants of Patient-Oncologist Prognostic Discordance in Advanced Cancer. JAMA Oncol. 2016 Nov; 2(11):1421-6.

19 Bernacki RE, Block SD; American College of Physicians High Value Care Task Force. Communication about serious illness care goals: a review and synthesis of best practices. JAMA Intern Med. 2014 Dec;174(12):19942003. 